\title{
DISCOVERY OF A NEW SOFT GAMMA REPEATER, SGR J1833-0832
}

\author{
E. GöĞÜşs ${ }^{1}$, G. Cusumano ${ }^{2}$, A. J. Levan ${ }^{3}$, C. Kouveliotou ${ }^{4}$, T. Sakamoto ${ }^{5}$, S. D. Barthelmy ${ }^{5}$, S. Campana ${ }^{6}$, Y. Kaneko ${ }^{1}$, \\ B. W. Stappers ${ }^{7}$, A. De Ugarte Postigo ${ }^{6}$, T. StrohmaYer ${ }^{5}$, D. M. Palmer ${ }^{8}$, J. Gelbord ${ }^{9}$, D. N. Burrows ${ }^{9}$, \\ A. J. van der Horst ${ }^{4,15}$, T. Muñoz-Darias ${ }^{6}$, N. Gehrels 5 , J. W. T. Hessels ${ }^{10,11}$, A. P. Kamble ${ }^{11}$, S. Wachter ${ }^{12}$, \\ K. WiERSEMA ${ }^{13}$, R. A. M. J. WiJers ${ }^{11}$, AND P. M. WoOds ${ }^{14}$ \\ ${ }^{1}$ Sabancı University, Orhanl1-Tuzla, 34956 İstanbul, Turkey; ersing@ @abanciuniv.edu \\ ${ }^{2}$ IASF-Palermo/Istituto di Astrofisica Spaziale e Fisica Cosmica di Palermo/Istituto Nazionale di Astrofisica (INAF), 90146 Palermo, Italy \\ ${ }^{3}$ Department of Physics, University of Warwick, Coventry CV4 7AL, UK \\ ${ }^{4}$ Space Science Office, VP62, NASA/Marshall Space Flight Center, Huntsville, AL 35812, USA \\ ${ }^{5}$ NASA/Goddard Space Flight Center, Greenbelt, MD 20771, USA \\ ${ }^{6}$ INAF-Osservatorio Astronomico di Brera, Via E. Bianchi 46, I-23807 Merate (LC), Italy \\ ${ }^{7}$ Jodrell Bank Centre for Astrophysics, School of Physics and Astronomy, The University of Manchester, Manchester M13 9PL, UK \\ ${ }^{8}$ Los Alamos National Laboratory, B-244, P.O. Box 1663, Los Alamos, NM 87545, USA \\ ${ }^{9}$ Department of Astronomy \& Astrophysics, The Pennsylvania State University, 525 Davey Lab, University Park, PA 16802, USA \\ ${ }^{10}$ Netherlands Institute for Radio Astronomy (ASTRON), Postbus 2, 7990 AA Dwingeloo, The Netherlands \\ ${ }^{11}$ Astronomical Institute, University of Amsterdam, Science Park 904, 1098 XH Amsterdam, The Netherlands \\ ${ }^{12}$ Spitzer Science Center/California Institute of Technology, Pasadena, CA 91125, USA \\ ${ }^{13}$ Department of Physics \& Astronomy, University of Leicester, Leicester, LE1 7RH, UK \\ ${ }^{14}$ Dynetics, Inc., 1000 Explorer Boulevard, Huntsville, AL 35806, USA \\ Received 2010 May 2; accepted 2010 May 17; published 2010 June 30
}

\begin{abstract}
On 2010 March 19, the Swift/Burst Alert Telescope triggered on a short burst with temporal and spectral characteristics similar to those of soft gamma repeater (SGR) bursts. The source location, however, did not coincide with any known SGR. Subsequent observations of the source error box with the Swift/X-Ray Telescope and the Rossi X-ray Timing Explorer led to the discovery of a new X-ray source with a spin period of $7.56 \mathrm{~s}$, confirming SGR J1833-0832 as a new magnetar. Based on our detailed temporal and spectral analyses, we show that the new SGR is rapidly spinning down $\left(4 \times 10^{-12} \mathrm{~s} \mathrm{~s}^{-1}\right)$ and find an inferred dipole magnetic field of $1.8 \times 10^{14} \mathrm{G}$. We also show that the X-ray flux of SGR J1833-0832 remained constant for approximately 20 days following the burst and then started to decline. We derived an accurate location of the source with the Chandra X-ray Observatory and we searched for a counterpart in deep optical and infrared observations of SGR J1833-0832, and for radio pulsed emission with the Westerbork Radio Synthesis Telescope. Finally, we compare the spectral and temporal properties of the source to other magnetar candidates.
\end{abstract}

Key words: pulsars: individual (SGR J1833-0832) - X-rays: bursts

Online-only material: color figure

\section{INTRODUCTION}

The first confirmed soft gamma repeater (SGR) burst was detected in 1979 but it took $\sim 7$ years (Laros et al. 1987; Atteia et al. 1987; Kouveliotou et al. 1987) to associate it with a newly discovered population of astronomical objects, namely, neutron stars repeatedly emitting soft and short gamma-ray bursts with peculiar properties. A decade later, these objects, together with their cousins, the Anomalous X-ray Pulsars (AXPs; see, e.g., Mereghetti 2008), were shown to possess extremely high magnetic fields $\left(\sim 10^{15} \mathrm{G}\right)$ as deduced from their spin periods and spindown rates, and thus were named magnetars (Duncan \& Thompson 1992; Thompson \& Duncan 1996; Kouveliotou et al. 1998; Vasisht \& Gotthelf 1997). The most distinguishing magnetar characteristics are a narrow range of spin periods (2-12 s), high spindown rates $\left(\sim 10^{-10} \mathrm{~s} \mathrm{~s}^{-1}\right)$, and unpredictable burst-active episodes. The latter can have a multitude of bursts with a wide luminosity range, anywhere from $10^{-2}$ to $10^{7}$ times the Eddington luminosity. These bursts result in the initial classification of a source as an SGR, which could then be subsequently confirmed as a magnetar with timing analysis (see

\footnotetext{
${ }^{15}$ NASA Postdoctoral Program Fellow.
}

Woods \& Thompson 2006 and Mereghetti 2008 for a detailed review on magnetars).

By 2008, a dozen sources were confirmed magnetars and a couple more were listed as magnetar candidates pending observations of new burst-active episodes. For the known sources, frequent spin period monitoring observations with the Rossi X-ray Timing Explorer (RXTE) over the last 15 years have provided a wealth of insight about their dynamics and energetics. Interestingly, despite their common characteristics, there are several important differences across this small population, and a larger sample of objects at different evolutionary stages is necessary to better understand the phenomenon. Magnetar hunting, however, is not an easy task; we have two strikes against us ab initio: their very small number and their very short active periods (days to months). Thus, the best way to discover magnetars is the combination of an all-sky X-ray and gammaray monitor to detect their bursts, and a timing instrument to measure their spin and spindown rates, and to estimate their magnetic fields.

On 2010 March 19, the Burst Alert Telescope (BAT) on board NASA's Swift satellite triggered at 18:34:50 UT on one short and soft burst (Barthelmy et al. 2010) originating from a region in the plane of our Milky Way galaxy that harbors at least three other magnetar candidates: AXP XTE J1810-197, and 


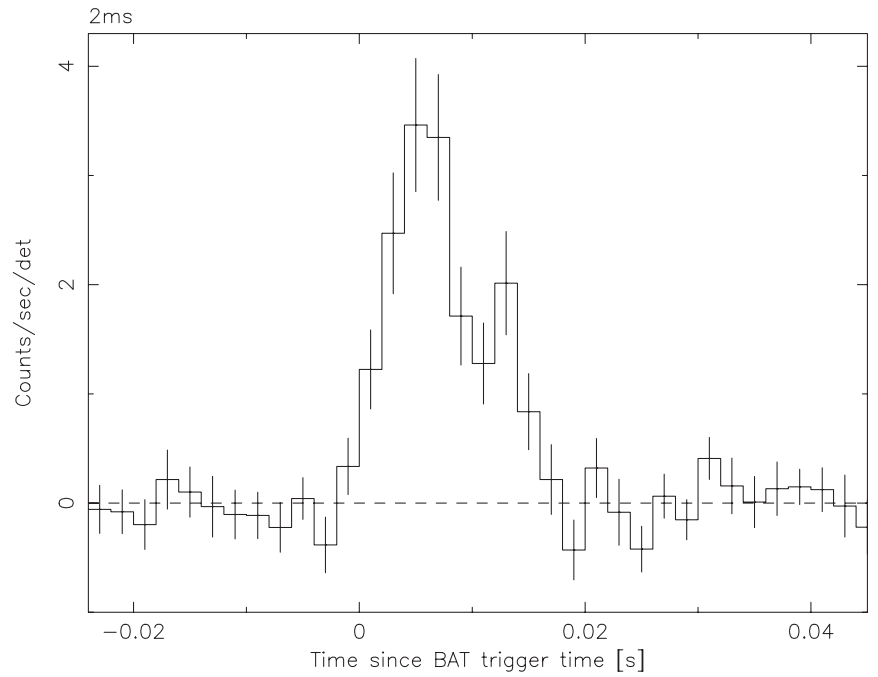

Figure 1. Swift/BAT light curve of the burst from SGR J1833-0832 in the $15-350 \mathrm{keV}$ band. The time bin size is $2 \mathrm{~ms}$.

SGRs 1806-20 and 1801-23. Using the initial source localization with the BAT, which had a $3^{\prime}$ error radius, we were able to exclude all these sources; further, based on the spectral and temporal properties of the event, we identified the source of the emission as a possible new SGR. Subsequent observations (starting from $60 \mathrm{~s}$ after the BAT trigger) of the source region with the Swift/X-Ray Telescope (XRT) provided the source position with a 1".6 uncertainty (Gelbord et al. 2010). Swift/Ultraviolet Optical Telescope (UVOT) observations starting $71 \mathrm{~s}$ after the trigger did not detect a source in the UV or optical bandpass at the XRT position (Marshall \& Gelbord 2010). To confirm the nature of the source, we observed it with $R X T E$ for $1.3 \mathrm{ks}$, less than $3.25 \mathrm{hr}$ after its discovery was reported. A subsequent timing analysis of this observation revealed coherent pulsations in the X-ray flux of the source with a period of $7.56 \mathrm{~s}$ (Göğüş et al. 2010). Thus, we were able to identify SGR J1833-0832 as a slowly rotating neutron star less than $6 \mathrm{hr}$ after its discovery, only the second time such a fast discovery was enabled due to the unprecedented synergy of Swift and RXTE (the first was the identification of SGR J0501+4516; Göğüş et al. 2008).

Swift was the only satellite that triggered on SGR J1833-0832; although the source was in the field of view (FOV) of the Gamma-ray Burst Monitor (GBM) on board the Fermi Gamma-ray Space Telescope (FGST), the fluence of the event was much lower than any of the GBM trigger thresholds. To precisely locate the source, we triggered our Target of Opportunity (ToO) observations with the Chandra X-Ray Observatory and obtained observations with the European Southern Observatory (ESO) Very Large Telescope/High Acuity Wide-field $K$-band Imager (VLT/HAWKI) and with the Gran Telescopio Canarias/ Optical Imager and Spectrograph (GTC/OSIRIS). We also observed with the Westerbork Synthesis Radio Telescope Pulsar Machine II (WSRT/PuMa II) and searched for radio pulsations from the source.

In this paper, we report on the discovery of SGR J1833-0832 and introduce its characteristics through our detailed multiwavelength investigations. In Section 2, we describe all multi-wavelength data used in our study. We present in Section 3 the precise localization of the new source using Chandra, Two Micron All Sky Survey (2MASS), and United Kingdom Infra-Red Telescope (UKIRT) data, and the tempo- ral and spectral characteristics of the persistent X-ray emission from the source using our Swift and RXTE observations. Finally, we discuss and interpret our results in Section 4.

\section{OBSERVATIONS}

\subsection{Swift/BAT}

Following the 2010 March 19 BAT discovery of a burst (trigger = 416485; Gelbord et al. 2010; Barthelmy et al. 2010), Swift slewed immediately to the onboard BAT position of R.A., decl. $=18^{\mathrm{h}} 33^{\mathrm{m}} 46^{\mathrm{s}},-08^{\circ} 32^{\prime} 13^{\prime \prime}\left(l, b=23^{\mathrm{h}} 19^{\mathrm{m}} 38^{\mathrm{s}}, 0^{\circ} 0^{\prime} 22^{\prime \prime}\right.$; radius uncertainty of $3^{\prime}, 90 \%$ containment). This location is 13.3 from the position of SGR 1806-20. The Swift/XRT detected a new X-ray source within the BAT error circle; these observations are described in Section 2.2. This was the only triggered burst detected from the source with BAT, and we report below on its spectral and temporal characteristics.

The burst was detected within the $87 \%$ BAT partial coding region; its mask-weighted light curve (Figure 1) shows a singlepeak structure. The $T_{90}$ duration (Kouveliotou et al. 1993) of the burst is $0.013 \pm 0.002 \mathrm{~s}(15-350 \mathrm{keV}$; total error including systematics).

We found that several continuum models could provide a statistically acceptable fit to the time-integrated spectrum $\left(T_{0}\right.$ to $T_{0}+0.016 \mathrm{~s}$ ): a power law with an exponential cutoff ${ }^{16}$ with a photon index, $\alpha$, of $-1.02 \pm 1.35$ and a $E_{\text {peak }}$ of $38.2 \pm 5.0 \mathrm{keV}$ $\left(\chi^{2} /\right.$ degrees of freedom $\left.(\mathrm{dof})=43.5 / 56\right)$; a blackbody with $k T_{\mathrm{BB}}=10.0 \pm 1.2 \mathrm{keV}\left(\chi^{2} / \mathrm{dof}=43.8 / 57\right)$; and an optically thin thermal bremsstrahlung ${ }^{17}$ with $k T_{\text {OTTB }}=29 \pm 5 \mathrm{keV}$ $\left(\chi^{2} /\right.$ dof $\left.=52.2 / 57\right)$. For the cutoff power-law model, the total fluence in the $15-150 \mathrm{keV}$ band is $(1.3 \pm 0.2) \times 10^{-8} \mathrm{erg} \mathrm{cm}^{-2}$ and the $6 \mathrm{~ms}$ peak flux (measured from $T_{0}+2$ to $T_{0}+8 \mathrm{~ms}$ ) is $(9.9 \pm 2.1) \times 10^{-7} \mathrm{erg} \mathrm{cm}^{-2} \mathrm{~s}^{-1}\left(19.4 \pm 3.8\right.$ photons $\left.\mathrm{cm}^{-2} \mathrm{~s}^{-1}\right)$. All quoted errors are at the $90 \%$ confidence level.

\subsection{Swift/XRT}

Swift/XRT observations of SGR J1833-0832 were obtained as a ToO monitoring program starting on 2010 March 19 at $\sim 10$ ks per observation for a total exposure of $\sim 184 \mathrm{ks}$ (Table 1 ). The XRT observations were coordinated with $R X T E$ in a manner that complemented and optimized usage of both instruments. The XRT data were accumulated in Windowed Timing (WT) mode only during the third observation (002; see Table 1), while all other data were collected in Photon Counting (PC) mode. In the WT mode, only the central $8^{\prime}$ of the FOV is read out, providing one-dimensional imaging and full spectral capability with a time resolution of $1.8 \mathrm{~ms}$. The PC mode provides full spatial and spectral resolution with a time resolution of $2.5 \mathrm{~s}$.

The data were processed with standard procedures (XRTPIPELINE v0.12.1) and filtering and screening criteria, using FTOOLS in the HEAsOFT package (v.6.6.1). We used a 0-2 and 0-12 grade selection for data in the WT and PC modes, respectively. This selection provides the best combination of spectral resolution and detection efficiency. SGR J1833-0832 was imaged far from the CCD hot columns so that no corrections inside the photon extraction regions were necessary.

We extracted the source events for timing and spectral analysis from a circular region of $47^{\prime \prime}$ radius (20 pixels) centered on the source position as determined with XRTCENTROID. The

\footnotetext{
16 Power law with an exponential cutoff is parameterized as $d N / d E=A(E / 50 \mathrm{keV})^{-\alpha} \exp \left(-E(2-\alpha) / E_{\text {peak }}\right)$.

17 OTTB is parameterized as $d N / d E=A(E / 50 \mathrm{keV})^{-1} \exp (-E / k T)$.
} 
Table 1

Summary of the Swift/XRT Observations

\begin{tabular}{lllr}
\hline \hline Seq. & \multicolumn{1}{c}{ Start Time (UT) } & \multicolumn{1}{c}{ End Time (UT) } & Exposure (s) \\
\hline 000 & 2010 Mar 19 18:35:50 & 2010 Mar 20 15:38:07 & 29174 \\
001 & 2010 Mar 21 04:14:14 & 2010 Mar 21 15:41:31 & 10756 \\
002 & 2010 Mar 21 16:47:37 & 2010 Mar 22 02:32:06 & 9954 \\
003 & 2010 Mar 22 07:50:12 & 2010 Mar 22 20:48:15 & 9869 \\
004 & 2010 Mar 23 08:58:47 & 2010 Mar 23 23:39:46 & 8068 \\
005 & 2010 Mar 24 01:07:40 & 2010 Mar 25 00:00:13 & 10266 \\
006 & 2010 Mar 25 01:36:34 & 2010 Mar 26 00:00:12 & 9844 \\
007 & 2010 Mar 26 12:28:42 & 2010 Mar 26 22:33:11 & 9961 \\
008 & 2010 Mar 27 10:57:33 & 2010 Mar 27 21:02:31 & 9855 \\
009 & 2010 Mar 28 00:04:08 & 2010 Mar 28 18:02:39 & 10919 \\
010 & 2010 Mar 29 12:44:13 & 2010 Mar 29 21:13:35 & 9511 \\
011 & 2010 Mar 31 00:20:50 & 2010 Mar 31 23:11:58 & 7942 \\
012 & 2010 Apr 3 14:59:40 & 2010 Apr 3 23:29:26 & 10028 \\
013 & 2010 Apr 7 13:45:23 & 2010 Apr 7 22:15:53 & 10097 \\
014 & 2010 Apr 12 09:41:24 & 2010 Apr 12 22:42:57 & 5130 \\
015 & 2010 Apr 13 03:08:11 & 2010 Apr 13 08:21:51 & 4054 \\
016 & 2010 Apr 15 00:01:07 & 2010 Apr 15 11:23:58 & 9417 \\
017 & 2010 Apr 18 14:53:02 & 2010 Apr 19 07:10:56 & 8817 \\
\hline
\end{tabular}

background was extracted from an annular region centered on the source with $90^{\prime \prime}$ and $120^{\prime \prime}$ internal and external radii, respectively. Ancillary response files were generated with XRTMKARF, and account for different extraction regions, vignetting, and point-spread function (PSF) corrections. We used the spectral redistribution matrices v011 in CALDB. All spectral fits were performed using XSPEC v.12.5.10 (Arnaud 1996).

\section{3. $R X T E$}

We monitored the source with the RXTE/Proportional Counter Array (PCA) from 2010 March 19, 21:49:10 UT (i.e., $\sim 3.25 \mathrm{hr}$ after the BAT trigger) to 2010 May 6 in 31 pointings. The time spacing between consecutive observations was on average 1.6 days, and ranged between 0.06 and 4.9 days. The exposure of individual observations varied between $1.3 \mathrm{ks}$ and $12 \mathrm{ks}$, with a total exposure of $178.6 \mathrm{ks}$ over $\sim 47$ days. In Table 2, we list a log of RXTE observations of SGR J1833-0832.

In this study, we employed PCA data collected in the 2-10 keV range. We inspected the light curve of each observation binned with $0.03125 \mathrm{~ms}$ to search for additional SGR bursts, and identified four events with duration $\lesssim 12 \mathrm{~ms}$. These events were rather weak compared to the typical bursts seen with the PCA from other SGRs; their detection indicates that a low level of bursting activity continued after the BAT trigger. We then filtered out the times of these four short bursts to obtain burst-free event lists for our timing studies. Finally, we converted all event arrival times to the solar system barycenter.

\subsection{Fermi/GBM}

Although GBM did not trigger on the Swift/BAT burst, we searched the daily GBM data (with time resolution of $256 \mathrm{~ms}$ in continuous mode and $64 \mathrm{~ms}$ in trigger mode) using our untriggered event search algorithm (Kaneko et al. 2010) to uncover any additional weak events from the source. The search was performed in the $10-300 \mathrm{keV}$ band on a 20 day-long segment starting from 2010 March 15 (i.e., four days prior to the BAT trigger). We did not find any untriggered events originating

Table 2

Summary of the RXTE Observations

\begin{tabular}{|c|c|c|c|}
\hline ObsID & Start Time (UT) & End Time (UT) & Exposure (s) \\
\hline $95048-03-01-10$ & 2010 Mar 19 21:49:10 & 2010 Mar 19 22:19:10 & 1335 \\
\hline $95048-03-01-00$ & 2010 Mar 21 08:07:28 & 2010 Mar 21 12:13:14 & 10443 \\
\hline 95048-03-01-01 & 2010 Mar 21 12:50:24 & 2010 Mar 21 15:22:10 & 6892 \\
\hline $95048-03-01-02$ & 2010 Mar 21 17:32:32 & 2010 Mar 21 23:13:10 & 13396 \\
\hline $95048-03-01-04$ & 2010 Mar 23 05:56:32 & 2010 Mar 23 11:03:10 & 11936 \\
\hline $95048-03-01-03$ & 2010 Mar 23 15:03:28 & 2010 Mar 23 19:08:10 & 9719 \\
\hline $95048-03-01-05$ & 2010 Mar 24 08:19:28 & 2010 Mar 24 09:17:10 & 3425 \\
\hline $95048-03-01-07$ & 2010 Mar 24 11:27:28 & 2010 Mar 24 12:19:10 & 3071 \\
\hline $95048-03-01-08$ & 2010 Mar 25 07:52:32 & 2010 Mar 25 10:21:10 & 6723 \\
\hline $95048-03-02-00$ & 2010 Mar 26 04:25:20 & 2010 Mar 26 06:49:10 & 6451 \\
\hline $95048-03-02-01$ & 2010 Mar 27 03:55:28 & 2010 Mar 27 06:21:10 & 6562 \\
\hline $95048-03-02-02$ & 2010 Mar 28 15:59:28 & 2010 Mar 28 18:18:08 & 5412 \\
\hline $95048-03-02-03$ & 2010 Mar 29 23:19:28 & 2010 Mar 30 01:39:10 & 5960 \\
\hline $95048-03-02-04$ & 2010 Mar 30 22:51:28 & 2010 Mar 31 01:02:10 & 5616 \\
\hline $95048-03-02-05$ & 2010 Apr 1 06:27:28 & 2010 Apr 1 07:12:10 & 2633 \\
\hline $95048-03-02-06$ & 2010 Apr 1 07:50:24 & 2010 Apr 1 08:45:10 & 3283 \\
\hline $95048-03-03-00$ & 2010 Apr 2 02:39:28 & 2010 Apr 2 05:10:08 & 6804 \\
\hline $95048-03-03-01$ & 2010 Apr 3 00:37:20 & 2010 Apr 3 01:34:10 & 3341 \\
\hline $95048-03-03-06$ & 2010 Apr 3 02:11:28 & 2010 Apr 3 03:08:10 & 3371 \\
\hline $95048-03-03-02$ & 2010 Apr 3 21:01:20 & 2010 Apr 3 23:32:10 & 6774 \\
\hline 95048-03-03-05 & 2010 Apr 6 19:38:24 & 2010 Apr 6 20:35:10 & 3321 \\
\hline $95048-03-04-00$ & 2010 Apr 9 02:33:20 & 2010 Apr 9 04:34:48 & 6810 \\
\hline 95048-03-04-01 & 2010 Apr 13 00:58:24 & 2010 Apr 13 03:01:10 & 5116 \\
\hline $95048-03-05-00$ & 2010 Apr 18 21:55:28 & 2010 Apr 19 00:26:10 & 6824 \\
\hline $95048-03-06-00$ & 2010 Apr 23 18:03:28 & 2010 Apr 23 20:35:10 & 6949 \\
\hline $95048-03-06-01$ & 2010 Apr 26 15:05:20 & 2010 Apr 26 15:53:10 & 2831 \\
\hline $95048-03-06-02$ & 2010 Apr 26 16:40:32 & 2010 Apr 26 17:36:10 & 3309 \\
\hline $95048-03-07-00$ & 2010 Apr 30 13:14:24 & 2010 Apr 30 15:45:10 & 6892 \\
\hline $95048-03-07-03$ & 2010 May 03 11:50:24 & 2010 May 03 12:46:08 & 3279 \\
\hline $95048-03-07-01$ & 2010 May 03 13:24:32 & 2010 May 03 14:21:10 & 3347 \\
\hline $95048-03-07-02$ & 2010 May 06 10:27:28 & 2010 May 06 12:58:10 & 6773 \\
\hline
\end{tabular}




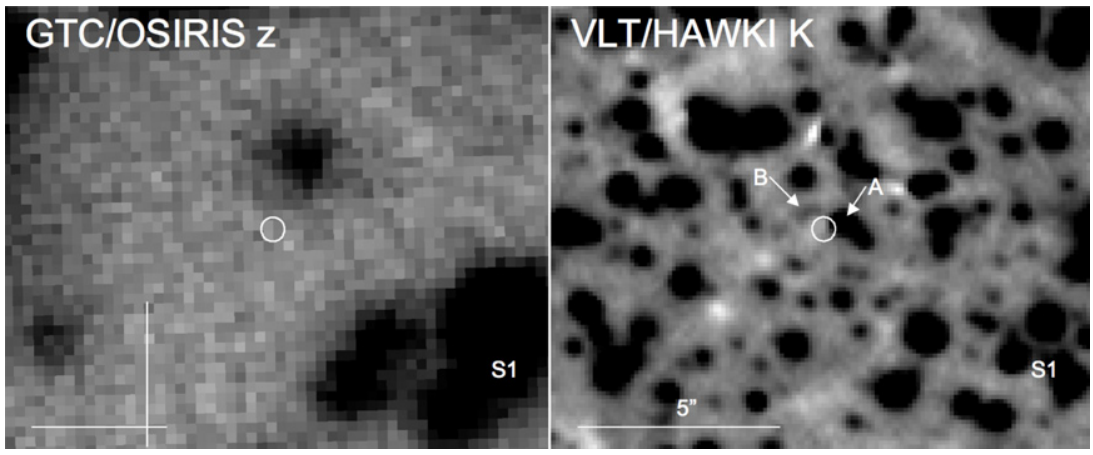

Figure 2. Zoomed-in region around SGR J1833-0832 in the GTC/OSIRIS image (left) and in the VLT/HAWKI image (right), showing the Chandra position (1 $\sigma$ error radius). Labels $A$ and $B$ indicate the objects close to the Chandra position of SGR J1833-0832 (see Section 3.1.2).

Table 3

X-ray Point Sources in the Chandra ACIS-I Field of SGR J1833-0832

\begin{tabular}{ccccc}
\hline \hline $\begin{array}{c}\text { Source } \\
\text { Name }\end{array}$ & $\begin{array}{c}\text { R.A. (J2000) } \\
\text { (hh mm ss.ss) }\end{array}$ & $\begin{array}{c}\text { Decl. (J2000) } \\
\text { (dd mm ss.ss) }\end{array}$ & $\begin{array}{c}\text { S/N } \\
\text { Ratio }\end{array}$ & $\begin{array}{c}\text { 2MASS } \\
\text { Association }\end{array}$ \\
\hline CXOU J183344.4-083108 & 183344.38 & -083107.71 & 53.7 & SGR, No \\
CXOU J183320.2-083426 & 183320.17 & -083426.34 & 6.1 & Yes \\
CXOU J183322.7-082409 & 183322.72 & -082408.89 & 6.5 & Yes \\
CXOU J183317.9-082516 & 183317.86 & -082515.45 & 6.1 & Yes \\
CXOU J183316.2-083615 & 183316.22 & -083615.22 & 8.7 & Yes \\
CXOU J183359.4-082229 & 183359.44 & -082229.18 & 6.4 & No \\
\hline
\end{tabular}

from the direction of SGR J1833-0832, including the BATtriggered burst and the bursts detected with $R X T E$. This is most likely due to the extremely short duration of the bursts $(\lesssim 16 \mathrm{~ms})$, which made them practically undetectable over the background with the coarse time resolution of the GBM continuous data. We also inspected the GBM data for the times of the four short and weak RXTE bursts and again found no evidence of activity.

\subsection{Chandra}

We initiated ToO observations with the Chandra Advanced CCD Imaging Spectrometer (ACIS-I) in the Timed Exposure (TE) mode on 2010 March 23 for an effective exposure of $33 \mathrm{ks}$. The source was relatively bright at the time of the ACIS-I observation, resulting in a (background-subtracted) count rate of $0.104 \pm 0.003$ counts $\mathrm{s}^{-1}(0.5-8 \mathrm{keV})$, which is expected to cause a pile-up at the $\sim 13 \%$ level. Our instrument choice was based on our goal, which was primarily to determine an accurate $\mathrm{X}$-ray position of the source, as described in Section 3.1. We selected data collected in the $0.5-8.0 \mathrm{keV}$ band with all four ACIS-I chips and generated a binned image (by a factor of 2) to improve the signal-to-noise ratio. We then employed wavdetect ${ }^{18}$ to search for sources with a detection threshold of $5 \sigma$ above the background and identified six uncataloged point sources, including SGR J1833-0832. We then repeated the same source finding procedure with an unbinned image to determine the accurate X-ray position of the SGR, which was at the aim point of ACIS-I. We list the X-ray positions (J2000) of all sources in Table 3. The last column in the table indicates whether an IR counterpart was identified for a source in the 2MASS data (see also Section 3.1).

\subsection{Optical and IR Observations}

The field of SGR J1833-0832 was observed with the imager OSIRIS, mounted on the $10.4 \mathrm{~m}$ GTC telescope, from

\footnotetext{
18 A source finding algorithm in CIAO 4.0.
}

Table 4

WSRT-PuMa II Observation Log

\begin{tabular}{cccc}
\hline \hline Epoch & MJD (day) & Duration $(\mathrm{hr})$ & Frequency $(\mathrm{MHz})$ \\
\hline 2010 Mar 23 & 55278.1570 & 1 & 2280 \\
2010 Mar 23 & 55278.1985 & 1 & 1380 \\
2010 Mar 24 & 55279.1962 & 1 & 1380 \\
2010 Mar 26 & 55281.2034 & 1 & 1380
\end{tabular}

2010 March 20 at 05:41:13 UT (i.e., $11.11 \mathrm{hr}$ after the BAT trigger). The observations were performed under non-optimal atmospheric conditions, with an average seeing of $\sim 1^{\prime \prime} .6$, and consisted of $20 \times 90 \mathrm{~s}$ long exposures of a $1.85 \times 2$. 35 portion of the sky using a Sloan $z$-band filter. The left panel of Figure 2 shows the combined GTC image of all 20 exposures of the field around SGR J1833-0832. The mean time of the observations is 06:04:04 UT on 2010 March 20. We did not detect any optical source within the Chandra error circle in the combined image down to a $3 \sigma$ limiting magnitude of 24.9.

We also observed the source using the ESO VLT/HAWKI in the $K_{\mathrm{s}}$ band under excellent seeing conditions (0.45). A total of 44 dithered exposures of $60 \mathrm{~s}$ duration were obtained, providing a total exposure time of $2640 \mathrm{~s}$. The mean time of the observations was 09:01:09 UT on 2010 March 25. There is no obvious IR source within the Chandra error circle; further astrometry and limiting magnitude information is given below (Section 3.1).

\subsection{WSRT/PuMa II Radio Observations}

We observed SGR J1833-0832 on three epochs in March, all within a week of the source discovery, using the WSRT at central frequencies of 2280 and $1380 \mathrm{MHz}$ (see Table 4 for details). The pointing position was R.A. $=18^{\mathrm{h}} 33^{\mathrm{m}} 44^{\mathrm{s}} 4$, decl. $=$ $-08^{\circ} 31^{\prime} 08^{\prime \prime}$. 4 . The WSRT was used in its tied-array mode, but as the position of the source was relatively uncertain, only telescopes out to baselines of $1.5 \mathrm{~km}$ were used. Furthermore, since one telescope was unavailable, only 11 telescopes were combined, giving a resolution of approximately $15^{\prime \prime}$ and $24^{\prime \prime}$ for 2280 and $1380 \mathrm{MHz}$, respectively, and a gain of $\sim 1 \mathrm{~K} \mathrm{Jy}^{-1}$. The WSRT splits the $160 \mathrm{MHz}$ bandwidth into eight $20 \mathrm{MHz}$ wide bands, and each band is recorded at baseband using the PuMa II pulsar back end (Karuppusamy et al. 2008). The baseband data were converted into filterbank format with 64 channels per $20 \mathrm{MHz}$ band and a sampling time bin of $8.192 \mathrm{~ms}$. The resulting 512-channel filterbank data were searched blindly for a periodicity matching the known period of SGR J1833-0832, and also folded using an ephemeris derived from the X-ray data 


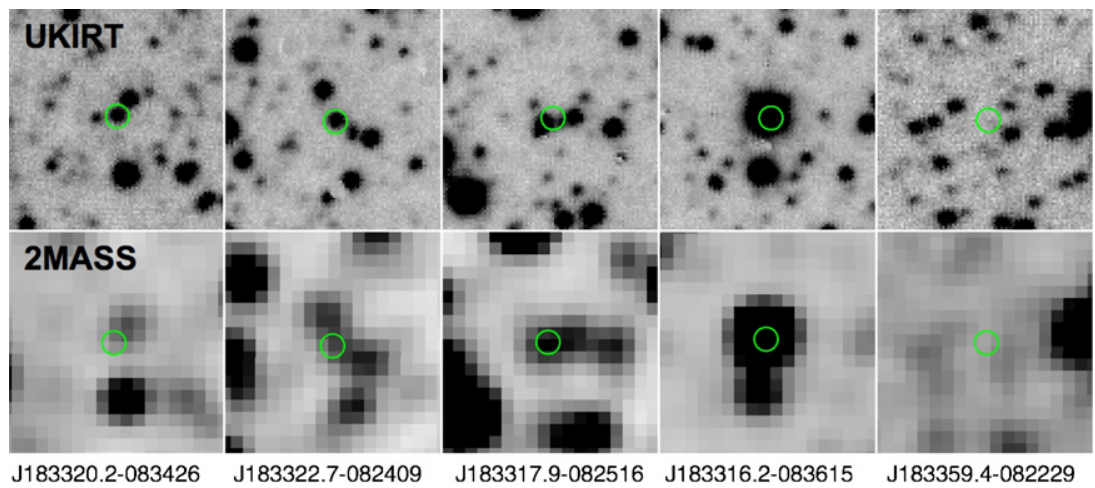

Figure 3. Chandra locations for the five X-ray sources overlayed on UKIRT images (top) and 2MASS images (bottom). As can be seen, the UKIRT allows an additional two counterparts to be identified (CXOU J183320.2-083426 and CXOU J183322.7-082409), which were ambiguous in the 2MASS data. (A color version of this figure is available in the online journal.)

(Section 3.2). We searched over a range of dispersion measures of $0-1800 \mathrm{pc} \mathrm{cm}^{-3}$, where the maximum is that expected along the line of sight through the Galaxy in the NE 2001 model (Cordes \& Lazio 2002). The long spin period of the source means that special attention had to be given to identify potential radio frequency interference. However, while there were some long-period features present in the data, they did not significantly affect our sensitivity to the source. Inspection of both the folded pulse profiles and the candidate lists from the blind searches did not reveal any significant detections of SGR J1833-0832. Using the radiometer equation (Dewey et al. 1985) and considering pulse widths of $50 \%$ and $10 \%$, which span the range of likely pulse widths for radio emitting magnetars, we obtain sensitivity limits of 0.6 and $0.2 \mathrm{mJy}$ at $2280 \mathrm{MHz}$ and 0.3 and $0.1 \mathrm{mJy}$ at $1380 \mathrm{MHz}$, corresponding to the $50 \%$ and $10 \%$ widths, respectively.

\section{DATA ANALYSES AND RESULTS}

\subsection{Precise Source Location and IR Counterpart Upper Limits}

\subsubsection{Imaging and Astrometry}

We searched the 2MASS archival data for IR counterparts of the five X-ray sources significantly detected in the Chandra image (Section 2.5), in addition to SGR J1833-0832 itself. To perform astrometry, we utilize observations taken as part of the UKIRT Deep Sky Survey (UKIDSS), which obtains deeper images than 2MASS (limiting magnitudes of $K \sim 18)$ with an improved PSF to aid with the elimination of source confusion. We show this improvement graphically in Figure 3, where we plot 2MASS and UKIRT images of each of the Chandra X-ray sources (excluding SGR J1833-0832, which is shown in Figure 2). We clearly see that one source, confused in the 2MASS images (CXOU J183320.2-083426), can be resolved by UKIRT; a second source (CXOU J183317.9-082516) remains apparently confused in the UKIRT images due to the contribution of a bright and faint source within the Chandra position. For the two remaining sources (CXOU J183320.2-083426 and CXOU J183316.2-083615), we confirm associations with 2MASS sources. To obtain astrometry of the field, we first aligned the UKIRT data to 2MASS, which gives a fit with an rms of $\sim 0^{\prime} .20$. Because of the source confusion in the 2MASS images at faint magnitudes, we limit ourselves to stars in the magnitude range $8<K<12$. We then compared the predicted locations of the $\mathrm{X}$-ray sources with their measured centroids, and fit the resulting data with a simple linear shift in $x$ and $y$ coordinates (since we do not have a large number of sources for comparison, this method provides a better estimate than allowing a larger number of free parameters). The resulting shifts are small: -0 '.25 in R.A. and 0 '. 2 in decl. The position of SGR J1833-0832, accurate to 0'.40, is R.A. $=18^{\mathrm{h}} 33^{\mathrm{m}} 44.37$, decl. $=-08^{\circ} 31^{\prime} 07^{\prime \prime} .5(\mathrm{~J} 2000)$.

This accuracy, however, is dominated by the error in the alignment of the UKIRT images to an absolute World Coordinate System (WCS; in this case 2MASS). A more accurate result was obtained by performing simple relative astrometry between the UKIRT and the Chandra images, providing a position accurate to $\sim 0$ '.3 (using the relative location of SGR J1833-0832 on the UKIRT frames). Although there is no obvious counterpart for SGR J1833-0832 in the UKIRT images, our deepest plausible limits come from our VLT/HAWKI observation with a total exposure of $2640 \mathrm{~s}$. The very small FOV of these observations precluded performing astrometry with Chandra sources directly (none of the five X-ray sources were in the HAWKI FOV). However, we could perform relative astrometry between the UKIRT and the VLT images with minimal error $(<0$ '.05), and hence place SGR J1833-0832 on the VLT/ HAWKI images with $\sim 0$ '.30 accuracy. The resulting location of SGR J1833-0832 is shown in Figure 2.

\subsubsection{IR Limits}

We do not detect a source within the $1 \sigma$ Chandra error circle of SGR J1833-0832 in the VLT/HAWKI images. Performing photometric calibration relative to 2MASS yields an upper limit for the SGR J1833-0832 counterpart within the Chandra/ ACIS-I error circle as $K_{\mathrm{s}}>22.4(3 \sigma)$. We should note, however, that this is an idealized scenario since confusion between faint sources is an issue at the lowest flux levels. While there are no sources within the $1 \sigma$ Chandra error radius, there are two sources at the $\sim 1.5 \sigma$ level from the Chandra position of SGR J1833-0832: a moderately bright source (marked A in Figure 2) with $K_{\mathrm{s}}=18.2 \pm 0.1$, and a fainter source (marked B) with $K_{\mathrm{s}}=21.2 \pm 0.2$. Since neither fall within the Chandra location, and due to the crowded nature of the field, we cannot make any strong claims for their association with SGR J1833-0832. Future observations to search for variability of these sources may provide a stronger diagnostic.

\subsection{Pulse Timing Analysis}

We used an epoch folding algorithm to determine the pulse ephemeris of SGR J1833-0832 as follows. We first determined 


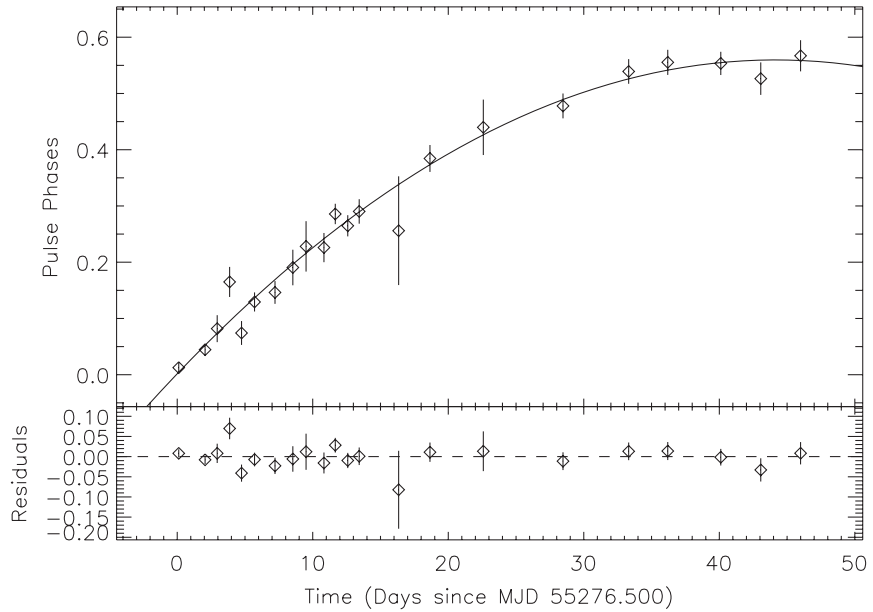

Figure 4. Top: plot of phase drifts for each segment of the PCA observations. The solid line is a second-order polynomial model for the phase drift in time; bottom: fit residuals.

the source pulse frequency using closely spaced $R X T E / P C A$ observations on 2010 March 21 with a total exposure of $44 \mathrm{ks}$ and generated a template pulse profile in the $2-10 \mathrm{keV}$ band. We then grouped all available PCA observations into 22 discrete time segments, each separated by at least 0.3 days. For each segment, we generated the pulse profile and cross-correlated it with the template to obtain the phase drift with respect to the template. We finally fit the phase shifts with a first- or higherorder polynomial to determine the pulse ephemeris of the source. The method summarized here is described in detail by Woods et al. (2002).

We first attempted to model the phase drifts with respect to the template using a first-order polynomial. This resulted in a poor fit $\left(\chi^{2} /\right.$ dof $\left.=122.1 / 21\right)$ with significant deviations of phase drift values from the model. Modeling the phase drifts with a secondorder polynomial results in a good fit $\left(\chi^{2} /\right.$ dof $\left.=19.9 / 20\right)$ with a spin period of 7.5654091(8) $\mathrm{s}$ and a period derivative of $(4.39 \pm 0.43) \times 10^{-12} \mathrm{~s} \mathrm{~s}^{-1}$ (epoch MJD 55276.5). We present in Figure 4 the pulse phase drifts with respect to the phase of the template profile with a second-order polynomial fit (top panel) and the residuals of the fit (lower panel).

\subsection{Pulsed Fraction and Pulsed Intensity History}

We investigated the energy dependence of the rms pulsed fraction $^{19}$ of SGR J1833-0832 by folding all available XRT data in PC mode in the $0.2-4 \mathrm{keV}, 4-6 \mathrm{keV}$, and 6-10 keV bands using the pulse ephemeris presented in Section 3.2. In Figure 5, we present the pulse profiles of SGR J1833-0832 in the three specified energy intervals (left) and the normalized profiles obtained by dividing with the maximum value of each pulse profile (right). We note that although the intensity of pulsations varies significantly in energy, the normalized amplitude of the pulsed signal remains remarkably constant. Consequently, we find that the rms pulsed fraction of SGR J1833-0832 does not vary as a function of energy with rms pulse fractions of $(34 \pm 2) \%,(36 \pm 2) \%$, and $(39 \pm 5) \%$ in the above energy intervals, respectively. Therefore, combining all available data

\footnotetext{
19 The rms pulsed fraction is defined as

$\mathrm{PF}_{\mathrm{rms}}=\left(\frac{1}{N}\left(\sum_{i=1}^{N}\left(R_{i}-R_{\mathrm{ave}}\right)^{2}-\Delta R_{i}^{2}\right)\right)^{\frac{1}{2}} / R_{\mathrm{ave}}$, where $N$ is the number of phase bins $(N=16), R_{i}$ is the rate in each phase bin, $\Delta R_{i}$ is the associated uncertainty in the rate, and $R_{\text {ave }}$ is the average rate of the pulse profile.
}

Table 5

Results of the Spectral Fits to the First 12 PC Mode Swift/XRT Observations

\begin{tabular}{lcccc}
\hline \hline \multicolumn{1}{c}{ Model } & $\begin{array}{c}\text { Column Density } \\
\left(10^{22} \mathrm{~cm}^{-2}\right)\end{array}$ & $\begin{array}{c}\Gamma / k T \\
\left(\mathrm{keV}^{-1}\right)\end{array}$ & $\begin{array}{c}2-10 \text { Unabs. (Abs.) Flux } \\
\left(10^{-12} \mathrm{erg} \mathrm{cm}^{-2} \mathrm{~s}^{-1}\right)\end{array}$ & $\begin{array}{c}\chi_{\text {red }}^{2} \\
(\mathrm{dof})\end{array}$ \\
\hline Power law & $16.4_{-1.0}^{+1.1}$ & $3.4_{-0.2}^{+0.2}$ & $17.3(4.0)$ & $1.03(143)$ \\
Blackbody $^{\mathrm{a}}$ & $9.9_{-0.7}^{+0.7}$ & $1.11_{-0.05}^{+0.05}$ & $7.4(3.8)$ & $1.06(143)$ \\
Bremsstrahlung $^{+0.0}$ & $13.7_{-0.7}^{+0.8}$ & $3.0_{-0.3}^{+0.4}$ & $11.6(3.9)$ & $1.01(143)$ \\
\hline
\end{tabular}

Note. ${ }^{a}$ The equivalent blackbody radius for a source at $10 \mathrm{kpc}$ is $R_{\mathrm{bb}}=$ $0.7 \pm 0.1 \mathrm{~km}$

in the $0.2-10 \mathrm{keV}$ range yields an rms pulsed fraction of $(36 \pm$ 2)\%, which also remained constant over the entire course of the $\sim 30$ days of XRT observations.

We also investigated the rms pulsed intensity variations of SGR J1833-0832 in time and energy using all available PCA data in the $2-4.5 \mathrm{keV}, 4.5-8.5 \mathrm{keV}$, and $8.5-14 \mathrm{keV}$ intervals. These energy bounds are selected to accommodate enough counts in each interval and determine the rms pulsed intensities with at least $5 \sigma$ significance. We find that the pulsed count rates of the source remain fairly constant in all three energy intervals at around $0.017,0.025$, and 0.006 counts $\mathrm{s}^{-1} \mathrm{PCU}^{-1}$, respectively.

\subsection{X-ray Spectral Properties}

We performed spectral analysis using only the XRT/PC observations, to avoid the high background effects of the WT observation. The data were rebinned to 20 counts per spectral bin to improve the fitting statistics. We fit the persistent source data with single-component models and found that the first 12 PC mode observations (i.e., observations 000 through 013 excluding 002 in Table 1) are fully consistent with a constant source. Consequently, we summed all 12 observations into one single spectrum, which contained $\sim 5000$ counts collected over $150 \mathrm{ks}$. We then rebinned the spectrum to 30 counts per bin and limited our spectral analysis to the $2-10 \mathrm{keV}$ energy range, to account for the high absorption toward the source direction, and fit several absorbed (using PHABS) single-component models within XSPEC. The best-fit spectral model parameters are listed in Table 5. Given the very high column density, the overall spectrum can be equally well fit by a steep power law $(\Gamma=3.4)$ or by a blackbody model with $k T \sim 1 \mathrm{keV}$; no additional components are required. We note that the derived neutral hydrogen column densities (see Table 5) are larger than the estimated Galactic column density of $1.7 \times 10^{22} \mathrm{~cm}^{-2}$ in the direction of SGR J1833-0832. When we fixed the column density to this Galactic value, no single- or double-component model was able to account for the observed spectrum, indicating that the high column density could be intrinsic to the source or to its surroundings. It is also important to note that the origin of the high column density might be high density of molecular hydrogen in the Galactic plane, on which the source is located.

We find evidence that the X-ray spectrum of SGR J1833-0832 hardens as its flux starts to decline in the last four PC mode observations (014 through 017 in Table 1): an absorbed power-law fit to these pointings yields indices in the interval from 2.6 to 3.2 , and a blackbody fit yields temperatures in the interval from 1.2 to $1.3 \mathrm{keV}$ (both at $90 \%$ confidence). The corresponding 2-10 keV unabsorbed (absorbed) power-law and blackbody fluxes are $12.1 \times 10^{-12}\left(3.5 \times 10^{-12}\right) \mathrm{erg} \mathrm{cm}^{-2}$ $\mathrm{s}^{-1}$ and $5.8 \times 10^{-12}\left(3.2 \times 10^{-12}\right) \mathrm{erg} \mathrm{cm}^{-2} \mathrm{~s}^{-1}$, respectively. 

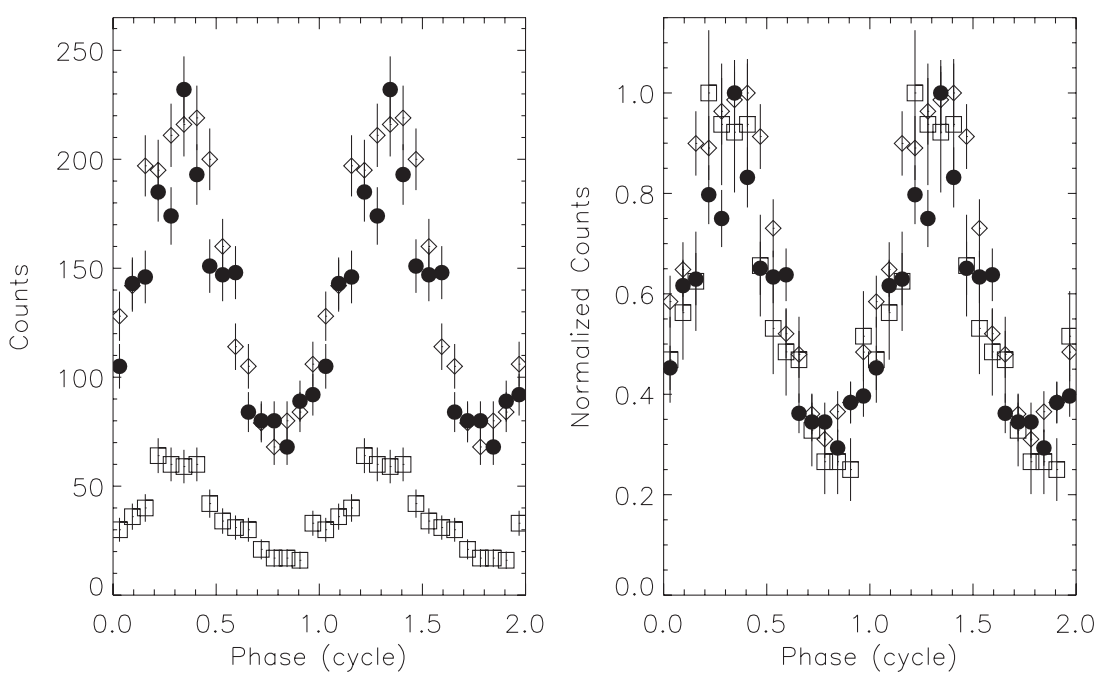

Figure 5. Left: pulse profiles of SGR J1833-0832 in the 0.2-4 keV (filled circles), 4-6 keV (diamonds), and 6-10 keV (squares) energy bands. Right: normalized pulse profiles to the peak value of each energy interval.

\section{DISCUSSION}

\subsection{Burst Emission}

At the onset of its active episode, SGR J1833-0832 emitted a single, relatively faint, burst that was seen with Swift/BAT and the International Gamma-Ray Astrophysics Laboratory (INTEGRAL; Kuiper \& Hermsen 2010); the total event fluence (15-350 keV) was $1.3 \times 10^{-8} \mathrm{erg} \mathrm{cm}^{-2}$. Compared to the persistent emission flux level $(2-10 \mathrm{keV})$, the burst energetics are very low: it would take only 13 minutes for the persistent source to emit an equivalent amount of energy. It is, therefore, unlikely that the (detected) burst emission provided the energy budget for the persistent X-ray emission.

The spectrum of the Swift/BAT burst is well described by a power law with exponential cutoff $\left(E_{\text {peak }}=38 \mathrm{keV}\right)$ or a blackbody with $k T=10 \mathrm{keV}$. Its spectral and temporal characteristics are very similar to those of known magnetars, in particular, to those of SGR J0418+5729, which emitted only two rather dim bursts in one day (van der Horst et al. 2010), and 1E 2259+586, which displayed a short episode of bursting for about $10 \mathrm{ks}$ in 2002 seen only in hard X-rays (Kaspi et al. 2003). In addition to the Swift/BAT event, we identified four weak X-ray bursts in our monitoring observations with the RXTE/PCA, which were not detected in the simultaneous Fermi/GBM data. It is interesting to note that the $R X T E / \mathrm{PCA}$ bursts are very similar in duration and X-ray intensity to the five short and rather weak bursts, also detected in the RXTE/PCA passband, emitted by the young, relatively strongly magnetized $\left(B_{\text {dipole }}=4.9 \times 10^{13} \mathrm{G}\right)$ rotation powered pulsar PSR J1846-0258 (Gavriil et al. 2008).

\subsection{Persistent Emission Properties}

The spin period, $7.5654091 \mathrm{~s}$, and period derivative, $4.39 \times$ $10^{-12} \mathrm{~s} \mathrm{~s}^{-1}$, of the source derived using the RXTE data are consistent (within 1.5 $\sigma$ ) with the values obtained by Esposito et al. (2010). Using these values, we estimate the dipole magnetic field strength of SGR J1833-0832 to be $B=$ $1.8 \times 10^{14} \mathrm{G}$, which is at the lower end of the $B$-fields of SGRs and close to the median value of those of AXPs $\left(B_{\text {median }}=2.2 \times 10^{14} \mathrm{G}\right)$. Note that the two recently discovered SGRs, J0418 +5729 and J0501 + 4516, possess the lowest
SGR surface magnetic fields of $<3 \times 10^{13} \mathrm{G}$ and $1.7 \times 10^{14} \mathrm{G}$, respectively.

Figure 6 exhibits the evolution of the persistent X-ray emission unabsorbed fluxes $(1-10 \mathrm{keV}$ ) for five magnetars (data obtained from Rea et al. 2009) and SGR J1833-0832, observed with Swift/XRT in the $2-10 \mathrm{keV}$ range. Each source was observed immediately after an outburst, when the persistent emission level had significantly increased from its quiescent state. We notice that all sources had similar maxima at the onset of their activation, ranging between $10^{-11}$ and $10^{-10} \mathrm{erg} \mathrm{cm}^{-2} \mathrm{~s}^{-1}$. Three of the sources (SGRs J1627-41, $\mathrm{J} 0501+4516$, and $\mathrm{J} 1900+14$ ) declined in a similar monotonic manner. However, SGR J1627-41 settled at the lowest flux level of all sources $\left(\sim 10^{-12} \mathrm{erg} \mathrm{cm}^{-2} \mathrm{~s}^{-1}\right)$, while SGR J1900+14 reached a flat plateau an order of magnitude higher and SGR J0501+4516 was still declining at the cessation of the XRT observations. The other two sources (1E 1547-5408 and CXOU J164710-455216) show trends similar to each other (but different from the other three sources): they remain flat after activation for 10-20 days and decline afterward with the same trend as the former three sources.

We have plotted the SGR J1833-0832 data as a band with an upper and lower bound on Figure 6; the upper flux limit is derived when we fit a single power law and the lower with a blackbody fit. Because of the excessive column density toward the source, there are very few photons below $2 \mathrm{keV}$ and the spectral fits are not well constrained; the real unabsorbed flux of the source is within these two bounds, and seems to remain constant over the first $\sim 20$ days following the onset of the outburst. Interestingly, we found an archival Chandra/ ACIS-S observation for a total of $8 \mathrm{ks}$ covering the field of SGR J1833-0832 starting on 2009 February 13 10:50:20 UT. The source was not detected within our Chandra error circle (Section 2.5), and we were able to derive a $2 \sigma$ upper limit (indicated by a dashed line on Figure 6) for its quiescent unabsorbed flux of $3.4 \times 10^{-13} \mathrm{erg} \mathrm{cm}^{-2} \mathrm{~s}^{-1}$ (Göğüş 2010). This is almost 100 times fainter than the current state of the source; assuming that SGR J1833-0832 reaches this level again, it would take at least two years if it follows the same decline trend of the other five sources as indicated by the last XRT observations. The source quiescent level is close to the lowest flux level of SGR 1627-41, the faintest magnetar thus 


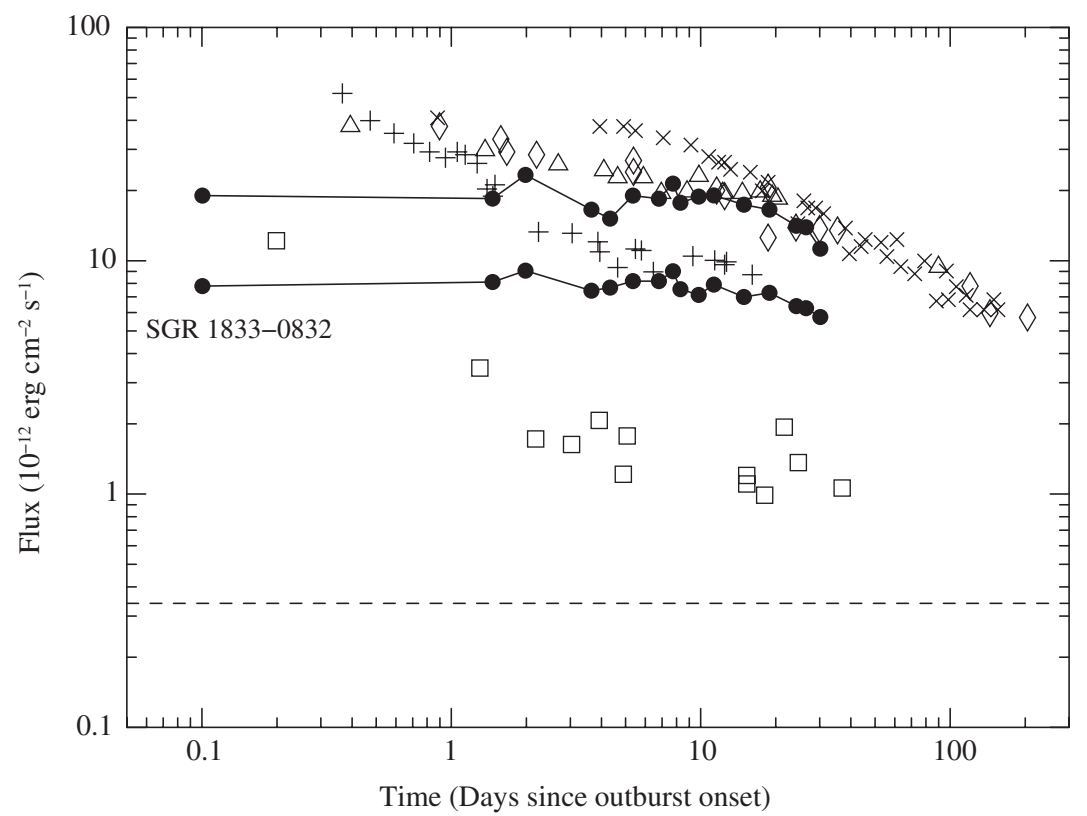

Figure 6. Unabsorbed flux of SGR J1833-0832 (filled circles), obtained by fitting power-law (upper values) and blackbody (lower values) models to XRT data in PC mode, compared with other sources (SGR 1900+14 (plus), 1E 1547.0-5408 (triangles), SGR 0501+4516 (cross), CXOU J164710-455216 (diamonds), and SGR 1627-41 (squares); data obtained from Rea et al. 2009). Time is measured from the onset of burst-active episodes. The horizontal dashed line indicates the Chandra $2 \sigma$ flux upper limit of SGR J1833-0832 from 2009 February 13.

far (Mereghetti et al. 2006; Esposito et al. 2009), and also to the flux of the faintest dim isolated neutron star RX J0420.0 - 5022 (Haberl et al. 2004), which is $4.8 \times 10^{-13} \mathrm{erg} \mathrm{cm}^{-2} \mathrm{~s}^{-1}$.

Finally, pulsed radio emission has been detected from two magnetars, XTE J1810-197 (Camilo et al. 2006) and 1E 1547.0-5408 (SGR J1550-5418; Camilo et al. 2007). The former showed radio pulsations after an X-ray outburst from the source, but the latter was seen in radio while the source was quiescent in X-rays. Searches for pulsed radio emission from other magnetars have been unsuccessful (e.g., Burgay et al. 2006; Crawford et al. 2007; Hessels et al. 2008). Our search for radio emission from SGR J1833-0832 with the WSRT resulted in non-detections, with upper limits of $0.2-0.6$ and $0.1-0.3 \mathrm{mJy}$ (depending on the assumed pulse widths) at 2280 and $1380 \mathrm{MHz}$, respectively. Burgay et al. (2010) reported an upper limit of $0.09 \mathrm{mJy}$ at $1400 \mathrm{MHz}$ on 2010 March 25 with the Parkes radio telescope. These upper limits are somewhat higher than some of the deepest radio searches for other magnetars, but more than an order of magnitude lower than the detections of XTE J1810-197 and 1E 1547.0-5408.

\subsection{Environment of SGR J1833-0832}

A visual inspection of the region surrounding SGR J1833-0832 on the $K_{\mathrm{s}}$-band images of the UKIRT GPS reveals no obvious source clustering in the vicinity of the source. However, as the field is small and crowded, low mass open clusters may be difficult to discern from bright background sources. We have performed a color-color search to uncover any cluster members; we caution, however, that it is still likely that the number of "unrelated" sources may obscure the detection of any faint, low surface brightness cluster that may be present.

There are several sources in the larger field which are plausible sites for the birth of SGR J1833-0832. One of these is the $\mathrm{H}$ II region IRAS $18310-0825$, at R.A. $=18^{\mathrm{h}} 33^{\mathrm{m}} 47^{\mathrm{s}} .9$, decl. $=-08^{\circ} 23^{\prime} 52^{\prime \prime}$, which is offset by roughly $7^{\prime}$ from SGR $\mathrm{J} 1833-0832$, another is [LPH96] 023.162+0.023 (R.A. = $18^{\mathrm{h}} 33^{\mathrm{m}} 23^{\mathrm{s}} .9$, decl. $=-08^{\circ} 40^{\prime} 32^{\prime \prime} ; 10$ arcmin offset, Lockman et al. 1996), while a third is [KC97c] G023.5+00.0 at R.A. = $18^{\mathrm{h}} 34^{\mathrm{m}} 20.0$, decl. $=-08^{\circ} 21^{\prime} 23^{\prime \prime} 7$, which is also associated with a cluster known as BDS2003 118 (Lockman 1986). Further, there are several dark clouds in the vicinity of SGR J1833-0832, which apparently do not host stellar populations yet. They have masses of $10^{2}-10^{3} M_{\odot}$, and are likely locations for a next generation of star formation within the region. Finally, there is also a known pulsar (PSR J1830 - 08) roughly 3.7 away from SGR J1833-0832 with an adopted distance of $5.67 \mathrm{pc}$ and a characteristic age of $\sim 147 \mathrm{kyr}$. It is possible that this source originates from the same star forming region associated with SGR J1833-0832.

While the dynamics of SGRs are as yet effectively unconstrained by observations (no SGRs have yet proper motion or radial velocity measurements), we can consider constraints by assuming that they (1) have a short lifetime $\left(<10^{4}\right.$ years), and (2) have a kick velocity distribution broadly comparable to that seen for young pulsars. In practice, this second assumption only improves the constraints we can make if the actual kicks are significantly smaller, and hence limit the transverse distance that SGR J1833-0832 could have traveled since birth. A typical pulsar kick might be of order $200 \mathrm{~km} \mathrm{~s}^{-1}$, so that in $10^{4}$ years the source may move $\sim 2 \mathrm{pc}$ on the sky. Hence, the angular scale SGR J1833-0832 could cover would be $\theta \approx 400^{\prime \prime} / d_{\mathrm{kpc}}$. For a distance of 6-12 kpc, this corresponds to an angular scale of $30^{\prime \prime}-70^{\prime \prime}$, although allowing for a range of kick velocities might extend this range by a factor of 3 or more. In this case, it would seem that none of the candidate clusters above are likely birthplaces. However, it is also possible (although not necessarily likely) that any progenitor may have "evaporated" from the cluster in which it formed with a velocity dispersion of a few $\mathrm{km} \mathrm{s}^{-1}$. With a lifetime of a few megayears, this may result in a progenitor at a large distance from the cluster, making our constraints weaker.

E.G. and Y.K. acknowledge EU FP6 Transfer of Knowledge Project "Astrophysics of Neutron Stars" (MTKD-CT-2006- 
042722). J.G. and D.N.B. acknowledge support from NASA contract NAS5-00136. A.J.v.d.H. was supported by an appointment to the NASA Postdoctoral Program at the MSFC, administered by Oak Ridge Associated Universities through a contract with NASA. C.K. acknowledges support by NASA grant NNH07ZDA001-GLAST. Partly based on observations made with the Gran Telescopio Canarias (GTC), installed in the Spanish Observatorio del Roque de los Muchachos of the Instituto de Astrofisica de Canarias, in the island of La Palma. The WSRT is operated by ASTRON (Netherlands Institute for Radio Astronomy) with support from the Netherlands foundation for Scientific Research. Partly based on observations made with ESO Telescopes at the La Silla or Paranal Observatories under program ID 084.D-0621.

\section{REFERENCES}

Arnaud, K. A. 1996, in ASP Conf. Ser. 101, Astronomical Data Analysis Software and Systems V, ed. G. Jacoby \& J. Barnes (San Francisco, CA: ASP), 17

Atteia, J.-L. 1987, ApJ, 320, L105

Barthelmy, S. D., et al. 2010, GCN Circ., 10528, 1

Burgay, M., Rea, N., Israel, G. L., Possenti, A., Burderi, L., di Salvo, T., D'Amico, N., \& Stella, L. 2006, MNRAS, 372, 410

Burgay, M., et al. 2010, ATel, 2515, 1

Camilo, F., Ransom, S. M., Halpern, J. P., \& Reynolds, J. 2007, ApJ, 666, L93

Camilo, F., Ransom, S. M., Halpern, J. P., Reynolds, J., Helfand, D. J., Zimmerman, N., \& Sarkissian, J. 2006, Nature, 442, 892

Cordes, J. M., \& Lazio, T. J. W. 2002, arXiv:astro-ph/0207156
Crawford, F., Hessels, J. W. T., \& Kaspi, V. M. 2007, ApJ, 662, 1183

Dewey, R. J., Taylor, J. H., Weisberg, J. M., \& Stokes, G. H. 1985, ApJ, 294, L25

Duncan, R. C., \& Thompson, C. 1992, ApJ, 392, L9

Esposito, P., et al. 2009, ApJ, 690, L105

Esposito, P., et al. 2010, ATel, 2550, 1

Gavriil, F., et al. 2008, Science, 319, 1802

Gelbord, J. M., et al. 2010, GCN Circ., 10526, 1

Göğüş, E. 2010, ATel, 2495

Göğüş, E., et al. 2008, GCN Circ., 8118, 1

Göğüş, E., et al. 2010, GCN Circ., 10534, 1

Haberl, F., et al. 2004, A\&A, 424, 635

Hessels, J., Rea, N., Ransom, S., \& Stappers, B. 2008, GCN Circ., 8134, 1

Kaneko, Y., et al. 2010, ApJ, 710, 1335

Karuppusamy, R., Stappers, B., \& van Straten, W. 2008, PASP, 120, 191

Kaspi, V., et al. 2003, ApJ, 588, L93

Kouveliotou, C., et al. 1987, ApJ, 322, L21

Kouveliotou, C., et al. 1993, ApJ, 413, L101

Kouveliotou, C., et al. 1998, Nature, 393, 235

Kuiper, L., \& Hermsen, W. 2010, ATel, 2509

Laros, J. G., et al. 1987, ApJ, 320, L111

Lockman, F. J., Pisano, D. J., \& Howard, G. J. 1996, ApJ, 472, 173

Lockman, F. J., et al. 1986, ApJS, 71, 469

Marshall, F. E., \& Gelbord, J. M. 2010, GCN Circ., 10540, 1

Mereghetti, S. 2008, A\&AR, 15, 225

Mereghetti, S., et al. 2006, A\&A, 628, 938

Rea, N., et al. 2009, MNRAS, 396, 2419

Thompson, C., \& Duncan, R. C. 1996, ApJ, 473, 322

van der Horst, A. J., et al. 2010, ApJ, 711, L1

Vasisht, G., \& Gotthelf, E. V. 1997, ApJ, 486, L129

Woods, P. M., \& Thompson, C. 2006, in Cambridge Astrophys. Ser. 39, Compact Stellar X-ray Sources, ed. W. H. G. Lewin \& M. van der Klis (Cambridge: Cambridge Univ. Press), 547

Woods, P. M., et al. 2002, ApJ, 576, 381 